\title{
BMJ Open Quality Improving transition outcomes in adolescents with permanent hearing loss
}

\author{
Chrysa Spyridakou, Surangi Mendis, Daniel DeVal
}

To cite: Spyridakou C,

Mendis S, DeVal D. Improving transition outcomes in adolescents with permanent hearing loss. BMJ Open Quality 2019;8:e000336. doi:10.1136/ bmjoq-2018-000336

Received 14 January 2018 Revised 6 August 2018 Accepted 19 February 2019

Check for updates

(C) Author(s) (or their employer(s)) 2019. Re-use permitted under CC BY-NC. No commercial re-use. See rights and permissions. Published by BMJ.

For numbered affiliations see end of article.

Correspondence to Dr Chrysa Spyridakou; chrysa.spyridakou@nhs.net
Young people with permanent hearing loss need to transition into adult services and engagement with the transition process is required to achieve this. Although there are clear national recommendations about the transition process and transfer to the adult services limited evidence has been published about the implementation and efficacy of transition programmes in young adults with permanent hearing loss. The primary aim of this quality improvement project was to significantly increase the number of teenagers with a clear transition protocol documented in their notes. The secondary aims were to ensure good medical and audiological outcomes based on all completing their medical investigations prior to transfer to the adult services and also based on a survey regarding attitudes to hearing aids use. A multiphase intervention programme was developed ; (1) staff update with national transition protocols, (2) development of a clear transition protocol, (3) allocated key worker (4) access and (5) engagement. Through these interventions we increased the number of teenagers who had a clear transition protocol documented in their notes from $11 \%$ to $91 \% .84 \%$ of our teenagers had completed their medical investigations, although documented discussion about the investigations had been done with $100 \%$ of the cohort. $9.2 \%$ of adolescents felt pressurised to use hearing aids compared to $35 \%$ prior to the interventions. This project resulted in clear, measurable conclusions and we have shown that we better understand the needs of our patients and the improvement is sustainable.

\section{PROBLEM}

This study deals with the transition of adolescents with permanent hearing loss from the paediatric to the adult audiovestibular medicine or ENT services. They are required to fully understand their condition in order to take responsibility for their health and continue to attend appointments as adults. Limited data have been published about the efficacy and outcomes of transition programmes in patients with hearing loss. However, National Institute for Health and Care Excellence (NICE) and the National Deaf Children's Society (NDCS) have provided guidance about the importance of clear transition pathways and their implementation. Transition protocols are widely accepted to be fundamental elements of good quality of care.
The Nuffield Speech and Hearing Centre is a large multidisciplinary tertiary unit. The majority of our adolescent patients have permanent hearing loss and wear their prescribed hearing aids. Patients who have received implantable devices (cochlear implants and bone anchored hearing aids) are seen in the department of the auditory implants and they were not included in this project. We aim to manage these patients holistically and address their medical, audiological, speech and language, psychological and educational needs. Although a specific departmental transition protocol had been a topic of the Multidisciplinary Team (MDT) discussions for some time, this had not been implemented prior to this quality improvement project.

Based on the above, there was need for a quality improvement project. The key aims were as follows:

- To significantly increase the number of teenagers with a clear transition protocol documented in the notes $(\geq 80 \%)$.

- To ensure good medical and audiological outcomes: aim for all $(100 \%)$ patients to have had their aetiological investigations completed and documented in the notes by the point of transfer to the adult services and repeat a survey about adolescents' attitudes towards hearing aids at the end of the project cycle.

\section{BACKGROUND}

The prevalence of permanent hearing loss at birth is $1.07 / 1000$ and this doubles to 2.05/1000 for children aged 9-16years. ${ }^{1}$ Hearing impairment is a substantial public health problem. It impacts on the life of the young person. Support from a multidisciplinary professional team is an essential requirement for successful lifelong management of the condition. Young people with permanent hearing loss need to transition into adult services, and engagement with the transition process is required to achieve this. 
Transition is defined in the Department of Health's 2006 publication 'Transition: Getting It Right for Young People' as

a purposeful, planned process that addresses the medical, psychosocial and educational/vocational needs of adolescents and young adults with chronic physical and medical conditions as they move from child-centred to adult-oriented health care systems. ${ }^{2}$

In February 2016, NICE published transition guidelines for young people who are long-term users of the health and social care services. There are clear recommendations about all stages of the process: the early and mid transition phases and the actual point of transfer to the adult services. $^{3}$

The NDCS published guidelines in 2011 specifically for the transition of deaf young adults based on two pilot projects in Devon and Tower Hamlets. Although clear transition guidelines exist, unfortunately, very little has been published about the effectiveness and outcomes of transition. ${ }^{4}$ The literature refers to the transition experience of adolescents who have received cochlear implants specifically. ${ }^{56}$ Issues about those patients receiving limited information and communication are highlighted.

Published evidence ${ }^{78}$ shows that adolescents can perceive use of hearing aids as 'being different' and stigmatising. Adolescents who perceive the use of hearing aids as normal are more likely to wear them. In a recent published study, ${ }^{8} 230$ adolescents aged $11-18$ years participated with a variety of hearing loss: $11 \%$ had mild hearing loss, $20 \%$ had moderate or moderate- severe, $41 \%$ had severe/profound and $28 \%$ had cochlear implants, and the perceived stigma domain scores were significantly lower (better) for the 11- 14 years age group than for the older participants aged $15-18$ years $(\mathrm{p}<0.0001$ and $\mathrm{p}=0.05)$.

There is evidence that the organisation of transition in health and social care is inconsistent. Professionals should work together with teenagers and their families to ensure that the transition process is a smooth and gradual one. Poorly managed transitions can result in disengagement with services and deterioration in health with poor outcomes for the young person. ${ }^{9}$ Perceptions of young people indicate that support focused on understanding their hearing loss and normalising the use of hearing aids should be a key feature of interventions.

\section{MEASUREMENT}

The first goal of this quality improvement project was to identify how many adolescents had a clear transition and transfer plan documented in their notes, in accordance with the departmental transition protocol (table 1). A consultant and a trainee doctor reviewed the notes and, using a checklist, ensured adherence to the departmental protocol for the respective age groups had been achieved.

From our electronic database, we identified 128 adolescents aged 13-18 years with permanent hearing loss who had been prescribed hearing aids. Details about the transition process had been documented in only 14 cases $(11 \%)$. Of these 14 cases, 12 were $>17$ years and due be transferred to the adult services, these 12 patients had

Table 1 Transition protocol

\begin{tabular}{|c|c|c|}
\hline $\begin{array}{l}\text { Early } \\
\text { transition } \\
\text { (age 13-14) }\end{array}$ & $\begin{array}{l}\text { AVP, audiologist, nurses, } \\
\pm \text { MDT (TOD, speech } \\
\text { and language therapist, } \\
\text { psychologist) }\end{array}$ & $\begin{array}{l}\text { Explain nature, degree and aetiology (where possible) of hearing loss to young } \\
\text { person and parents/guardians } \\
\text { Review hearing aids } \\
\text { Define goals in each of the above competency areas } \\
\text { Work through each area } \\
\text { Agreement on each action taken } \\
\text { Develop personal health record book/folder } \\
\text { Discuss role of key worker } \\
\text { Agree next appointment }\end{array}$ \\
\hline $\begin{array}{l}\text { Middle } \\
\text { transition } \\
\text { (age 15-16) }\end{array}$ & $\begin{array}{l}\text { AVP, audiologist, nurses, } \\
\pm \text { MDT (TOD, speech } \\
\text { and language therapist, } \\
\text { psychologist) }\end{array}$ & $\begin{array}{l}\text { Review clinical progress } \\
\text { Audiological assessment } \\
\text { HA assessment } \\
\text { Competency-based assessments } \\
\text { Patient questionnaire } \\
\text { Action plan based on above assessments } \\
\text { Ensure patient knows the key worker } \\
\text { Referral to transfer service discussed, and information leaflet given } \\
\text { Visit the adult services if transfer is planned internally and if the young person } \\
\text { wishes }\end{array}$ \\
\hline $\begin{array}{l}\text { Transfer } \\
\text { (age 17-18) }\end{array}$ & $\begin{array}{l}\text { AVP, audiologist, nurses, } \\
\pm \text { MDT (TOD, speech } \\
\text { and language therapist, } \\
\text { psychologist) }\end{array}$ & $\begin{array}{l}\text { Review clinical progress } \\
\text { Audiological assessment } \\
\text { Review role of key worker } \\
\text { - Visit the adult services if transfer is planned internally } \\
\text { Summarise medical and audiological history if transfer is planned externally }\end{array}$ \\
\hline
\end{tabular}

AVP, audiovestibular physician; HA, hearing aid; MDT, multidisciplinary team; TOD, teachers of the deaf. 
clear documentation about preparation for the transfer. In the remaining two cases, who were both under the age of 17 , there was only brief documentation about transition in the notes.

Additionally, a patient satisfaction survey carried out in 2016 noted that overall satisfaction with the services at the Nuffield Speech and Hearing centre was high among teenagers $(>90 \%)$ but that $35 \%$ reported feeling pressurised to wear their hearing aids. We felt that a repeat survey regarding adolescents' attitudes towards hearing aids should be an aim of this project as hearing aid use is regarded as a positive predictor of successful transition based on current published evidence.

The aim of this project was to ensure that a clear transition/transfer protocol exists and to ensure that all professionals adhere to it when it has been documented in the notes.

To ensure that the transition protocol improved the overall quality of care, we selected the following as measurable outcomes based on current evidence: (1) completion of the medical investigations to ascertain the cause of the patient's hearing loss, the outcomes of which should be documented appropriately in the notes;
(2) repeat the survey regarding adolescents' attitudes towards hearing aids with the aim of increasing the rate of satisfaction with The Nuffield services and reducing the number of patients feeling pressurised to wear their amplification.

\section{DESIGN}

The next step was to identify areas where intervention was required to improve the quality of care. It was clear that this required a multitargeted approach. A multidisciplinary team was formed consisting of a consultant audiovestibular physician, the lead paediatric audiologist, a senior paediatric nurse and a specialist teacher for hearing impairment. Input was also received from a specialist speech and language therapist and a clinical psychologist. Five primary areas of intervention were identified: staff awareness, the presence of a clear transition protocol, the requirement for an allocated key worker, patient access and patient engagement (figure 1).

Staff were updated on the latest transition guidelines and were shown how to identify adolescents who were
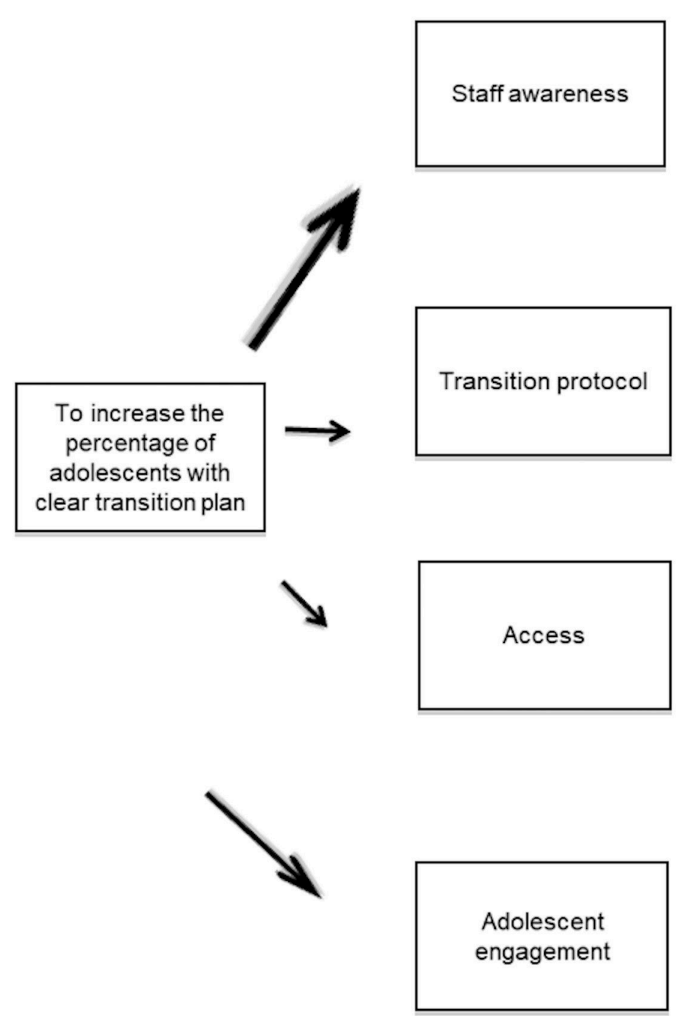
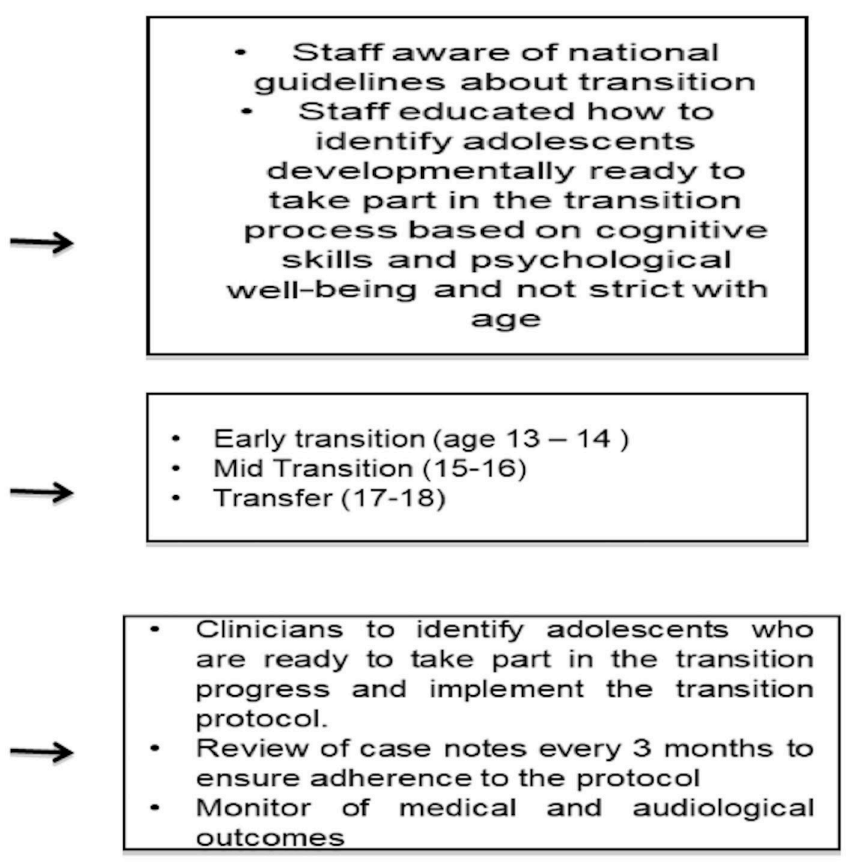

- Documentation in the notes regarding adolescents and carers understanding about transition

- Repeat survey at the final stages of the project regarding attitudes of adolescents regarding hearing aids

Figure 1 Expected BMJ Quality Improvement Project outcomes. 


\section{Percentage of adolescents with clear documentation of transition/ transfer pathway}

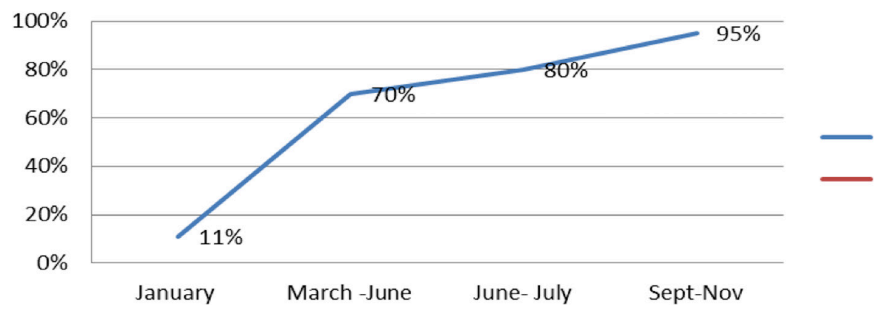

Figure 2 Percentage of adolescents with clear documentation of their transition pathway.

developmentally and psychologically ready to take part in transition planning.

The key professionals met once every 4 weeks initially to develop the transition protocol (table 1) and implement it. Subsequently, the clinic notes were reviewed every 3 months to ensure that the protocol was being adhered to and to also monitor the outcomes. Finally, a survey was completed at the end of the project to look for changes in adolescents' attitudes towards their hearing aids.

\section{STRATEGY}

Our Specific Measurable Achievable Realistic Time-limited aim was to increase the percentage of patients who are reviewed by an MDT and have a clear transition plan documented in the notes to $>80 \%$ by November 2017 .

\section{PDSA (Plan-Do-Study-Act) cycle 1 (December 2016 to January 2017)}

The first step was to identify the key professionals required and the different stages of the transition process. This was achieved by the MDT professionals meeting regularly to review the key stages of transition and decide what each stage would involve.

We identified three stages of transition, starting from the age of 13 years, in accordance with the NDCS and NICE guidelines (table 1). The key outcomes for each phase were to ensure that the young person and their carer(s) understood the transition process, understood their hearing loss and knew how to use their amplification devices and were aware of the medical investigations that had been undertaken to establish the cause of their hearing loss. If the patient and their carer(s) were agreeable, the medical investigations would be completed prior to transfer of the patient to the adult services and should be clearly documented in the notes.

Although the transition process usually commences at the age of 13, staff were informed that this age is not rigid. Transition does not start at the same age for everyone but depends on the cognitive ability and psychological wellbeing of the adolescent.
PDSA cycle 2 (February 2017)

Following development of the departmental transition protocol, we realised that a key worker had not been allocated to each of our adolescents with permanent hearing loss. According to 2016 NICE transition guidelines, the named key worker should be someone the young person knows well and feels comfortable with. It was agreed by all professionals that this role would be undertaken by the senior paediatric audiologists at The Nuffield.

\section{PDSA cycle 3 (March 2017 to November 2017)}

The next intervention cycle involved implementation of the transition pathway and dissemination of information about the key worker's role. Adolescents attending the consultant clinics were identified prospectively. Adolescents in the early, mid and transfer stages of the transition process were seen in accordance with the protocol and the discussion and agreed plan were fully documented in their corresponding clinic letter. Those who were ready to be transferred to the adult services were given the option of either attending their local audiology/ENT service or transferring internally to our adult Audiovestibular Medicine team. If they chose to attend their local service, a full summary of their medical, audiological and other needs was transferred to the local services. Patients identified in this cycle were entered into a database and the consultant in charge of their care reviewed their notes every 3 months to monitor adherence to the transition protocol and also monitor their medical and audiological outcomes.

\section{PDSA cycle 4 (August 2017 to November 2017)}

A survey was conducted during this period to monitor quality improvement following the implementation of the transition protocol. Based on the results of the initial patient satisfaction survey in 2016, when a significant number of adolescents reported feeling pressurised to wear their hearing aids, a follow-up survey was designed. Adolescents aged 13-18 were interviewed to establish (1) if they wear their hearing aids, (2) if so, for how long, (3) in what situations do they wear their aids and (4) who facilitates their use. If they did not wear their amplification, we wanted to establish what the barriers to wearing them were and if parents, teachers or healthcare professionals were perceived to be pressurising the adolescents into wearing their hearing aids.

\section{RESULTS}

Each of the PDSA cycles was implemented over a 2-month period.

From 1 March 2017 to 30 November 2017, the case notes of the adolescents ranging from 13 to 18 years were reviewed by the consultant audiovestibular physician and trainee doctor in audiovestibular medicine every 3 months and the following were noted using a checklist:

- If the transition process was discussed with the patients and their carer(s). 
A

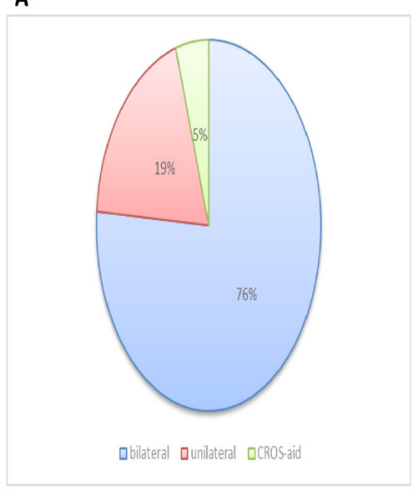

B

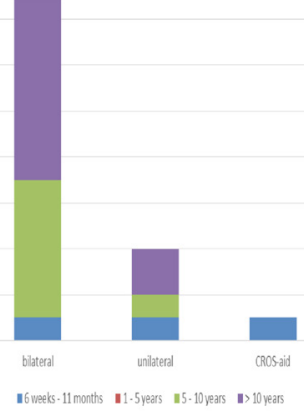

Figure 3 (A) Type of amplification devices used by those surveyed (bilateral behind-the-ear aids, unilateral behind-theear aid or a CROS-aid). (B) Length of time patients had been wearing their hearing aids for CROS, contralateral routing of signals.

- If key elements of each transition phase (early, mid, transfer) had been discussed.

- If the adolescents and their families were aware of who their key worker was and how to contact them.

- How long the young person wore their hearing aids for each day.

- Which medical investigations had been performed and whether the outcomes had been conveyed.

Between 1 March 2017 and 30 November 2017, a total of 68 adolescents were seen at the consultant's clinics. 23 were in the 'early transition' age bracket, 23 were in the 'mid transition' age bracket and 15 were at the stage of transfer to the adult services. 56 adolescents had bilateral hearing loss and 12 unilateral hearing loss (seven left sided and five right sided). Of the adolescents with bilateral hearing loss, 11 had mild to moderate hearing loss, eight mild to severe and five mild to profound. Sixteen had bilateral moderate hearing loss, 10 moderate to severe and two moderate to profound. Four had bilateral severe hearing loss. Four used a contralateral routing of signals (CROS) hearing aid, nine had a left post aural hearing aid, five had a right post aural hearing aid and 50 had bilateral post aural hearing aids.

One hundred per cent had an audiological assessment at the time of their appointment and had the opportunity to liaise with the audiology team if they had queries with regards to their hearing aids but 20 out of the 68 $(29.5 \%)$ did not have formal joint appointments with the audiologist for a full hearing aid review. However, for those 20 patients, joint appointments were subsequently requested. 62 of the 68 patients $(91 \%)$ had a stable hearing loss with no additional medical or rehabilitation concerns and were therefore reviewed annually.

Following implementation of the transition pathway, there was an initial improvement in rates of documentation of the transition information in the notes from $11 \%$ to $70 \%$. We noted a subsequent further increase to $95 \%$ in the latter stages of the data collection process (figure 2). This related to education of staff, increased awareness of the transition pathway and improved documentation.

Regarding the secondary aims of this quality project, 57 out of the $68(84 \%)$ adolescents had completed their aetiological investigations and 31 out of $68(45.5 \%)$ had a known cause of their hearing loss. Nine out of 11 who had not completed of their medical investigations agreed to be referred for further investigations. Only two adolescents (aged 13 and 15) were not keen to have further tests as they found them to be invasive previously, for example, MRI and blood tests. These two patients wished to defer their further investigations to a later date.

The survey of attitudes of adolescents towards their hearing aids interviewed 21 patients (mean 14.8 years): one patient had been prescribed a CROS aid $(5 \%)$, four unilateral hearing aids (19\%) and 16 bilateral (76\%). Ninety per cent wear them at school and $42 \%$ wear them at school and at home. Children are more likely to wear their hearing aids in environments they and their parents consider to be important. Children with mild to moderate hearing loss do not feel that they need to wear the hearing aids at home.

Only two out of the $21(9.5 \%)$ felt pressurised by teachers and healthcare professionals to wear their hearing aids compared with the previous study of $35 \%$, which is a significant improvement. 13 out of $21(62 \%)$ reported that they do not need any parental encouragement to wear their amplification devices, four out of the $21(19 \%)$ said they needed occasional parental encouragement and four out of 21 (19\%) needed constant parental encouragement. Those who did not wear their hearing aids did not approve of their cosmetic appearance (figure 3).

\section{LESSONS AND LIMITATIONS}

This quality improvement project highlights the need for a clear multidisciplinary transition pathway for adolescents with permanent hearing loss. Transition is a complex process and having a clear pathway in place ensures that healthcare professionals adhere to national guidelines and regularly monitor their practice. It clarifies the roles and responsibilities of clinical members of staff and actively engages the adolescent and their carer(s) with the process.

Having a clear transition pathway is a mandatory requirement and an indication of good quality service. It ensures successful transition to adult services and successful lifelong management of the hearing loss. Additionally, involving adolescents in their care and covering all the key aspects (eg, medical, audiology, education and overall well-being) avoids duplication and unnecessary follow-up appointments. Prior to this project, patients were followed up in the medical clinic after 6-12 months. However, for adolescents who were seen between March 2017 and November 2017, full discussion of the transition 
process by senior and junior clinicians had been documented in the notes and clinic letters, and there was a clear management plan in place and therefore they could be reviewed every twoyears in the medical clinic. We are therefore in the process of developing a new review pathway.

Only some aspects of the transition process were analysed by this pilot project. For example, it is important to establish whether or not these young adults actually attend their appointment(s) in the adult services as they require support, particularly in the first six months after they are first seen in the adult department. The majority of our patients choose to be transferred internally and therefore this would be straightforward to monitor. We would need to work closely with our colleagues in the adult services for this to take place. It would also be prudent to re-analyse patient satisfaction following the actual transfer process.

This quality improvement project has not included the adolescents who did not attend their appointments with us, and therefore, may not be representative of all adolescents with hearing loss. We have also not included our deaf adolescents with learning disabilities or complex medical needs in this project as we feel that a different transition pathway should be followed for such patients.

\section{CONCLUSION}

This quality improvement project aimed to implement national transition guidelines for long-term conditions, specifically for adolescents with hearing loss. Current practice was audited and compared with national standards. This project resulted in the development of a departmental transition pathway, which the relevant clinicians have familiarised themselves with. Adherence to this protocol has shown significant improvement in our outcomes, and although this project began 10 months ago, we strongly believe that we can maintain these excellent outcomes with further improvements. This project resulted in clear, measurable conclusions, and we have shown that we better understand the needs of our patients.

The next step would be to reduce the number of medical appointments for our adolescents, as $84 \%$ of them have completed their medical investigations and $91 \%$ have a stable hearing loss. Such patients can be reviewed in audiologist-led clinics and only require a review in the medical clinics by the audiovestibular physician at the key transition phases, for example, on transfer to the adult services.
We also plan to follow up the adolescents who do not attend for their planned appointments via a telephone survey to identify how we can facilitate their attendance.

We will present the project outcomes at our national audit meeting and share our practice and experiences with our colleagues.

Author affiliations

Audiovestibular Medicine, Royal National Throat Nose and Ear Hospital, London, UK

Acknowledgements Mr Azhar Shaida, consultant ENT surgeon and Dr Breege Mac Ardle, consultant Audiovestibular Physician

Contributors CS designed, analysed and wrote the paper. SM carried out the adolescents survey, analysed the results and contributed to the final version of the paper. DDV provided the data (list of adolescents with hearing loss) of the original audit.

Funding This quality improvement project was given the divisional Royal National Throat, Nose and Ear Hospital license in January 2017 by CAQIC.

Competing interests None declared.

Patient consent for publication Not required.

Provenance and peer review Not commissioned; externally peer reviewed.

Open access This is an open access article distributed in accordance with the Creative Commons Attribution Non Commercial (CC BY-NC 4.0) license, which permits others to distribute, remix, adapt, build upon this work non-commercially, and license their derivative works on different terms, provided the original work is properly cited, appropriate credit is given, any changes made indicated, and the use is non-commercial. See: http://creativecommons.org/licenses/by-nc/4.0/.

\section{REFERENCES}

1. Fortnum HM, Summerfield $A Q$, Marshall $D H$, et al. Prevalence of permanent childhood hearing impairment in the United Kingdom and implications for universal neonatal hearing screening: questionnaire based ascertainment study. BMJ 2001;323:536-40.

2. Department of Health. Transition: getting it right for young people, 2006.

3. National Institute for Health and Care Exellence. NICE Guidelines Transition from children's to adults' services for young people using health or social care services, 2016.

4. National Institute for Health and Care Exellence. NDCS Transition from paediatric to adult audiology services: Guidelines for professionals working with deaf children and young people, 2011.

5. Olds J, Fitzpatrick EM, Séguin C, et al. Perspectives of young people and their parents in the transition of cochlear implant services: implications for improved service delivery. Cochlear Implants Int 2014;15:2-12.

6. Olds J, Fitzpatrick E, Séguin C, et al. Facilitating the transition from the pediatric to adult cochlear implant setting: perspectives of $\mathrm{Cl}$ professionals. Cochlear Implants Int 2012;13:197-205.

7. Kent B, Smith S. They only see it when the sun shines in my ears: exploring perceptions of adolescent hearing aid users. J Deaf Stud Deaf Educ 2006;11:461-76.

8. Patrick DL, Edwards TC, Skalicky AM, et al. Validation of a quality-oflife measure for deaf or hard of hearing youth. Otolaryngol Head Neck Surg 2011;145:137-45.

9. Department of Health. Children and young people's health outcomes strategy, Report of the children and young people's health outcomes forum, 2012 